\title{
A cluster of two human cases of tick-borne encephalitis (TBE) transmitted by unpasteurised goat milk and cheese in Germany, May 2016
}

SO Brockmann ${ }^{1}$, R Oehme ${ }^{2}$, T Buckenmaier ${ }^{3}$, M Beer $^{4}$, A Jeffery-Smith 5 , M Spannenkrebs ${ }^{6}$, S Haag-Milz ${ }^{7}$, C Wagner-Wiening ${ }^{2}$, C

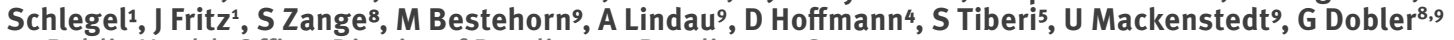

1. Public Health Office, District of Reutlingen, Reutlingen, Germany

2. State Health Office Baden-Württemberg, Stuttgart, Germany

3. Veterinary Health Office, District of Reutlingen, Reutlingen, Germany

4. Friedrich-Loeffler Institute, Federal Institute of Animal Health, Institute of Diagnostic Virology, Greifswald, Insel Riems, Germany

5. Barts Health NHS Trust, London, United Kingdom

6. Public Health Office, District of Biberach, Biberach, Germany

7. Public Health Office, District of Sigmaringen, Sigmaringen, Germany

8. Bundeswehr Institute of Microbiology; German Consultant Laboratory of TBE, German Center of Infection Research (DZIF), Munich, Germany

9. Parasitology Unit, University of Hohenheim, Stuttgart, Germany

Correspondence: Gerhard Dobler (gerharddobler@bundeswehr.org)

Citation style for this article:

Brockmann SO, Oehme R, Buckenmaier T, Beer M, Jeffery-Smith A, Spannenkrebs M, Haag-Milz S, Wagner-Wiening C, Schlegel C, Fritz J, Zange S, Bestehorn M, Lindau A, Hoffmann D, Tiberi S, Mackenstedt U, Dobler G. A cluster of two human cases of tick-borne encephalitis (TBE) transmitted by unpasteurised goat milk and cheese in Germany, May 2016. Euro Surveill. 2018;23(15):pii=17-00336. https://doi.org/10.2807/1560-7917.ES.2018.23.15.17-00336

In May 2016, two cases of tick-borne encephalitis (TBE) were confirmed by serology (positive IgM and IgG antibodies against TBE virus (TBEV) in serum), with a possible link to raw milk and cheese from a goat farm in a region in Baden-Württemberg, Germany not previously known as TBE-endemic. The outbreak investigation identified 32 consumers of goat dairy products (29 consumers, one farm employee, two owners) of whom none had IgM antibodies against TBEV 3-8 weeks after consumption. Of the 27 notified TBE cases in the State, none reported consumption of raw goat milk or cheese from the suspected farm. Five of 22 cheese samples from 18 different batches were RT-qPCR-positive for TBEV -genome, and two of the five samples were confirmed by virus isolation, indicating viability of TBEV in the cheese. Nine of the 45 goats had neutralising TBEV antibodies, two of them with a high titre indicating recent infection. One of 412 Ixodes ricinus was RT-qPCR-positive, and sequencing of the $E$ gene from nucleic acid extracted from the tick confirmed TBEV. Phylogenetic analyses of tick and cheese isolates showed $100 \%$ amino acid homology in the $E$ gene and a close relation to TBEV strains from Switzerland and Austria.

\section{Introduction}

Tick-borne encephalitis (TBE) is caused by an RNA virus of the genus Flavivirus, family Flaviviridae [1]. There are at least three known subtypes and so far, only the European subtype has been detected in various central European countries (Austria, Czech Republic,
France, Germany, Slovak Republic, Switzerland) in ticks, rodents and patients since the 1950s [2]. TBE virus (TBEV) circulates between ticks and natural hosts (small mammals) in geographically strictly limited natural foci. TBE in animals is not well understood yet and the knowledge on the pathogenesis of TBEV in domestic animals is limited [3]. However, symptomatic infection has been reported in dogs, monkeys and horses, and seroconversion without specific neurological signs of TBE has been described in ruminants such as cattle, goats and sheep $[4,5]$.

TBE in humans is the most important tick-borne viral disease in Europe and Asia, and up to 10,000 human cases are reported annually, although it is assumed that many mild and subclinical infections remain undiagnosed and both the infection and the disease are highly under-reported [6]. The main infection route of TBEV is by tick bite. However, food-borne transmission via untreated dairy products is a known way of transmission of TBEV. Milk-borne transmission for instance was common in the Czech Republic in the 1950s, and the related disease was called 'biphasic milk fever' [7]. Since then, milk-borne TBE outbreaks have been reported in a number of central and eastern European countries [8-13]. One TBE outbreak caused by goat cheese happened in 2008 in a mountainous region in Austria [14].

In Germany, TBE has been a reportable disease since 2001 , with some 200 to 400 human cases reported 


\section{FIGURE 1}

Investigation of a food-borne tick-borne encephalitis cluster Baden-Württemberg, Germany, May-July 2016 $(\mathrm{n}=2)$

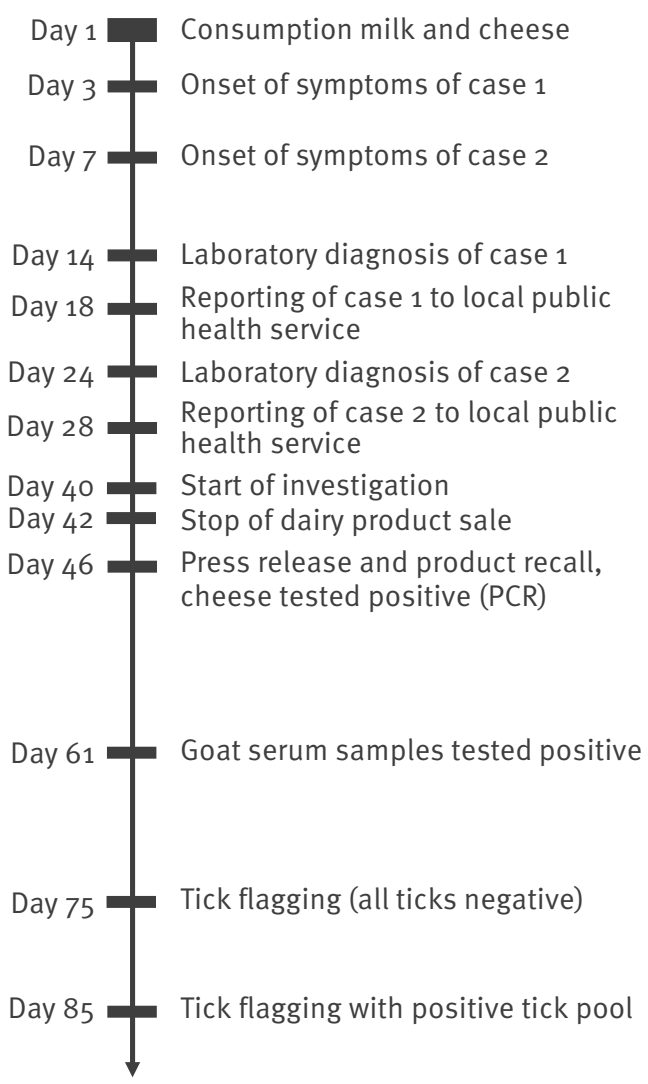

annually [15]. Most human cases in Germany appear in southern Germany, where the main known endemic areas are located. Only about half of the patients in Germany remember tick bites as the probable cause of the TBEV infection. However, so far, there has not been any evidence of milk-borne TBEV infection in Germany.

We investigated a cluster of two human TBE cases that occurred in the spring/summer of 2016 in southern Germany in consumers of raw goat milk and cheese from a goat farm. After the clinical diagnosis of meningoencephalitis and serological confirmation as TBE in both cases, the local public health office was informed and an outbreak investigation was initiated on 14 June in order to identify the source of infection and the extent of the outbreak. The following points were addressed: identification of additional human cases, identification of the natural focus of TBEV on the goat farm, seroprevalence of anti-TBEV antibodies in goat sera on the farm and isolation and characterisation of TBEV from goat cheese and goat milk as well as ticks sampled around the farm.

\section{Methods}

\section{Human infections}

For active case finding among the consumers, information and an alert were released on 20 June 2016 by the local government through the regional local print media and by the manufacturer for their clients. The local public health office offered serological testing against TBEV by the German TBE consultant laboratory to all consumers who could be retrospectively identified. A human case was defined as exhibiting positive results in IgM and IgG against TBEV in association with the consumption of goat milk or cheese from the affected goat 4 four weeks before to 4 weeks after the first case was diagnosed and having no vaccination against TBE within the 6 months before. To exclude false positive results caused by cross-reactivity to other flaviviruses, all sera were tested for IgM and IgG antibodies against different flaviviruses (TBEV, West Nile virus, dengue virus, yellow fever virus, Japanese encephalitis virus) using an indirect immunofluorescence assay (Euroimmun, Lübeck, Germany), according to standard procedures. The consumers were asked for the date of the farm visit or cheese purchase, consumption date and total consumed amount, if available.

The regional physicians' council was informed about suspected food-borne TBEV infections in the region. The Baden-Wuerttemberg State Health Office contacted all local health offices in the State to re-investigate already notified TBE cases with date of onset of clinical symptoms in May and June 2016 (calendar week 17-25), with special regard to consumption of raw goat milk products in the outbreak region within 21 days before disease onset.

\section{Goat cheese}

The responsible food safety authority sampled unpasteurised goat cheese from the manufacturing and storage facilities of the dairy farm (16 June), and consumers provided available residual samples of cheese and milk produced between 5 May to 15 June by the goat farm. A $10 \%$ suspension in cell culture medium (Minimal Essential Medium, Invitrogen, Karlsruhe, Germany) was prepared and centrifuged and the 'milk serum', the watery phase, was used for nucleic acid extraction and further analyses. PCR-positive cheese samples were suspended in cell culture medium and incubated on $80 \%$ confluent Vero cells and A549 cells according to standard procedures of the Bundeswehr Institute of Microbiology. The cell culture supernatants were harvested on day 7 after inoculation and nucleic acid was extracted using the viral RNA extraction kit (Qiagen, Hilden, Germany). The extracted nucleic acids were tested by RT-qPCR according to a published protocol [16]. The E genes of TBEV were sequenced by Sanger sequencing as described in Kupca et al. [17].

\section{Goat flock}

All goats in the flock were born on the farm. According to the owner's information, no external animals from 
Phylogeny of the E genes of a tick-borne encephalitis virus isolated from goat cheese compared with related and reference TBEV strains, Baden-Württemberg, Germany, $2016(\mathrm{n}=22)$

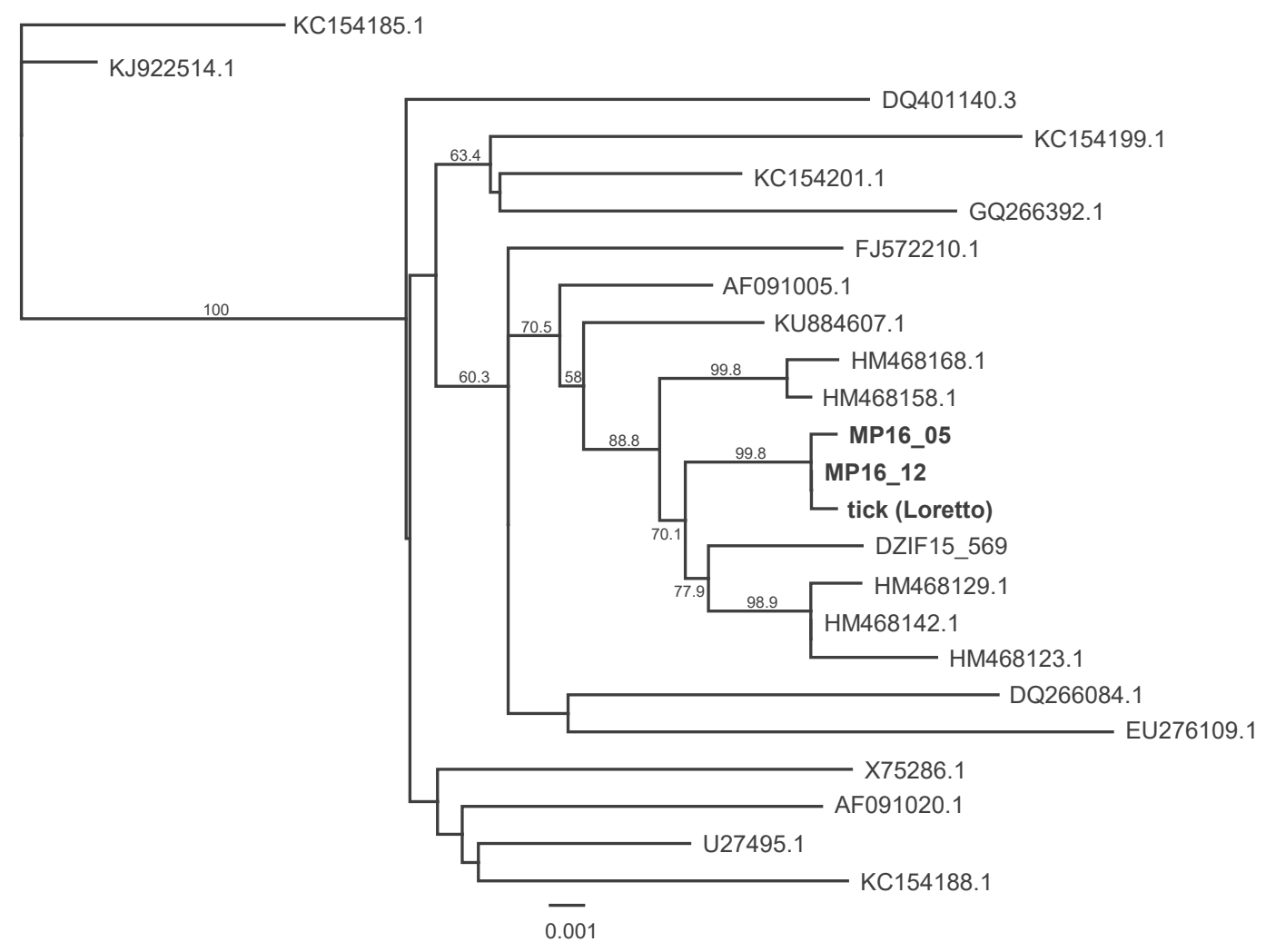

Sequences were chosen to include the reference strains Neudörfl and Hypr and the genetically closely related strains to demonstrate the genetic relatedness to the strains. E gene sequence accession numbers and origin of isolates: TBEV strain MP16 05 (GenBank accession

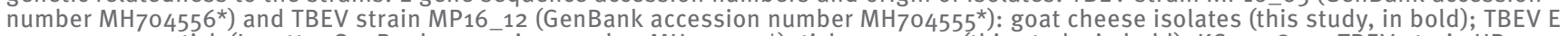
gene sequence tick (Loretto, GenBank accession number MH704557*): tick sequence (this study, in bold); KC154185.1: TBEV strain HB17111 (Germany 2011); KJ922514.1: TBEV strain Skrivanek (Czech Republic, 1953); DQ401140.3: TBEV strain Torö (Sweden, 2003); KC154199.1: TBEV strain A104 (Austria, 1990); KC154201.1: TBEV strain CG-223 (Slovak Republic, 1990); GQ266392.1: TBEV strain AS33 (Germany 2005); FJ572210.1: TBEV strain Salem 2008); AFo91005.1: TBEV strain Absettarov (Russia, 1951); KU884607.1: TBEV strain Leila-BH95/15 (Germany, 2015); HM468168: TBEV strain UR Schattdorf1 (Switzerland, 2010); HM468158.1: TBEV strain TG Lommis1 (Switzerland, 2010); DZIF 15_569: TBEV strain Pitztal (Austria, 2015); HM468129.1: TBEV virus strain AG Zofingen (Switzerland, 2010); HM168142.1: TBEV strain LU Dagmarsellen (Switzerland, 2010); HM468123.1: TBEV strain AG Brittnau (Switzerland, 2010); DQ266084.1: TBEV strain 263/5 (Czech Republic, 1991); EU276109.1: TBEV strain KrM93 (South Korea, 2006); X25286.1: TBEV strain Hypr (Czech Republic, 1953); AFo91020.1: virus strain ZZ9 (Austria, 1985); U27495.1: TBEV strain Neudörfl (Austria, 1971); KC154188.1: TBEV strain HM377-11 (Germany, 2011).

other farms had been added to the flock during the 2 years before the incident. For analysis of the goats' serostatus and to find infected goats, we took a blood sample from each animal in the goat flock and tested it by virus neutralisation test at the German Federal Institute of Animal Health (Friedrich Loeffler-Institute, Greifswald-Insel Riems, Germany), using the low-pathogenic TBE group virus Langat virus according to standard procedures [18]. All sera were also tested for TBEV RNA by RT-qPCR [16] to demonstrate a potential acute viraemia in the animals.

\section{Ticks and natural foci}

Because the meadows the goats used for grazing during the time before the outbreak (between April and May 2016) were documented by the farm owner, ticks were sampled around all suspected meadows between July and September 2016 by flagging along the vegetation. Ticks were identified and pooled according to stage and sex (three to 10 nymphs and two to five adult females or males per pool, all larvae in one pool). Pools were crushed using a MM400 mixer mill (Retsch, Germany) and three steel beads per pool, and nucleic acid was extracted using the EasyMag system (Biomerieux, Germany). Extracted nucleic acid was tested for TBEV RNA by a RT-qPCR [16].

\section{Results}

\section{Human infections}

The two cases who developed TBE after the consumption of raw milk and cheese from the goat farm in May 2016 were not vaccinated (Figure 1). The first case was a man who presented to hospital in May 2016 with fever, headache and difficulty to walk, 3 days after returning from a hiking holiday in south-west Germany with three other participants (one man, two women). They had visited a dairy farm where the first case and 
TABLE

Goat milk products tested for the presence of tick-borne encephalitis virus, Baden-Württemberg, Germany, May to June $2016(\mathrm{n}=23)$

\begin{tabular}{|l|c|c|c|}
\hline $\begin{array}{l}\text { Food item } \\
\text { (unpasteurised) }\end{array}$ & $\begin{array}{c}\text { Production } \\
\text { date }\end{array}$ & PCR & Culture \\
\hline Goat milk & 16 Jun 2016 & Negative & ND \\
\hline Goat cream cheese & 15 Jun 2016 & Negative & ND \\
\hline Goat cream cheese & 14 Jun 2016 & Negative & ND \\
\hline Goat cream cheese & 13 Jun2016 & Negative & ND \\
\hline Goat cream cheese & 12 Jun2016 & Positive & Positive \\
\hline Goat cream cheese & 11 Jun2016 & Negative & ND \\
\hline Goat cream cheese & 10 Jun2016 & Positive & Positive \\
\hline Goat cheese, ripened & 9 Jun2016 & Positive & Negative \\
\hline Goat soft cheese & 8 Jun2016 & Positive & Negative \\
\hline Goat soft cheese & 5 Jun2016 & Negative & ND \\
\hline Goat soft cheese & 6 Jun2016 & Negative & ND \\
\hline Goat cheese, ripened & 4 Jun2016 & Negative & ND \\
\hline Goat cream cheese & Jun 2016 & Positive & Negative \\
\hline $\begin{array}{l}\text { Goat cream cheese with } \\
\text { curry }\end{array}$ & Jun 2016 & Negative & ND \\
\hline Goat soft cheese & Jun 2016 & Negative & ND \\
\hline Goat soft cheese & Jun 2016 & Negative & ND \\
\hline Goat soft cheese & May 2016 & Negative & ND \\
\hline Goat soft cheese & May 2016 & Negative & ND \\
\hline Goat soft cheese & May 2016 & Negative & ND \\
\hline Goat soft cheese & May 2106 & Negative & ND \\
\hline Goat soft cheese & May 2016 & Negative & ND \\
\hline Goat soft cheese & 3 May 2016 & Negative & ND \\
\hline Goat soft cheese & April 2016a & Negative & ND \\
\hline
\end{tabular}

ND: not done.

a Stored cheese with unknown production date.

b Selling day at the dairy farm.

one other member of the group consumed unpasteurised goat milk and unpasteurised goat cheese. The second case was the other man from the same group who presented to hospital 4 days later with neurological symptoms.

During the course of illness, both cases developed various neurological symptoms and suffered from severe fatigue and weight loss. Both were admitted to hospital and treated for meningoencephalitis with empirical antibiotic and anti-viral therapy. Other potential causes were excluded by a number of different blood tests, cerebrospinal fluid tests and magnetic resonance imaging. TBEV serology was positive for IgM and IgG in the serum and cerebrospinal fluid (CSF) for both cases (day 14, day 24) (Figure 1). The clinical symptoms improved, but both continued to experience asthenia, neurological and psychiatric symptoms after 1 year. The final outcome is unfortunately unknown.

The two other hiking group members did not consume any milk and only one woman tried small amounts of the goat cheese but disgorged it immediately. None of the two women developed any neurological symptoms, nor IgM or IgG antibodies against TBEV. The farm owner reported more than 20 visitors/consumers on the assumed day of infection. Farm visitors consumed ca 20-30 glasses of raw milk and a much larger amount of cheese products. However, no further cases were reported from this potential exposure. After the second reported TBE case from the same family the district public health office started an investigation on day 40 resulting in a stop of the farm's dairy product sale and all dairy products still available were recalled on day 42 (Figure 1). On day 46, the goat cheese samples tested positive and after two tick sampling activities on days 75 and 85 , a positive tick pool was detected by PCR in the samples from day 85 (Figure 1 ).

None of 29 other consumers in June who responded to the press release of the manufacturer and the local government on day 46 after start of symptoms of the first human case (Figure 1) nor the dairy worker showed IgM antibodies against TBEV 3-8 weeks after consumption. Four consumers had low anti-TBEV IgG titres (without cross-reactions to other tested flaviviruses), which indicate either TBE vaccination or past infection. The dairy worker had a high IgG titre but reported no TBE vaccination and no TBE-specific symptoms in the months before the event. The person remembered only a few tick bites in their life. As the dairy worker reported daily consumption of self-produced cheese but did not drink any goat milk, their serological result may be interpreted as a potential recent infection through cheese, although we cannot exclude an infection through ticks in the TBE focus area. The farm owner had been vaccinated against TBE early in the season 2016 and was therefore not tested. None of the 27 TBE cases notified until June 2016 in the State reported consumption of raw goat milk or cheese or a visit to the suspected farm; they were therefore not considered to be related to the outbreak.

\section{Dairy products}

We tested 22 cheese samples and one milk sample from 18 different batches (production days) in May and June 2016 for the presence of TBEV RNA. Five samples from five different batches of cheese (cream cheese, soft cheese and ripened cheese) produced between 8 June and 12 June tested positive by repeated RT-qPCR. Two of the five samples could be confirmed by cell culture, indicating the viability of TBEV in the cheese (Table).

We retested by TBEV RT-qPCR the five PCR-positive cheese samples each divided into 10 portions of ca $1 \mathrm{~g}$. Three and two portions from the two culture-confirmed samples were weakly positive, while the remaining 45 samples were negative, indicating inhomogeneous distribution of virus in the cheese samples.

The five positive samples were inoculated in cell cultures. Two of the samples yielded a virus isolate in Vero 
and A549 cells. Sub-passages were performed and the $E$ genes of the isolated virus strains were amplified and sequenced. Both isolates had 100\% identical nucleotide sequences for the $\mathrm{E}$ genes. Using the maximum likelihood method, the closest phylogenetic relation was found to the $E$ genes of a TBEV strain we isolated from a tick pool in 2015 in Pitztal, Austria, and to published sequences from different locations in Switzerland (Zofingen, Dagmarsellen, Brittnau, Lommis, Schattdorf) (Figure 2) [19].

\section{Goat flock}

Blood was taken from all 45 goats at the beginning of June 2016 and tested for TBE antibodies. The animals were 1-11 years-old. Nine goats had neutralising antibody titres $>15$ against TBEV, which proved that these animals had been infected with TBEV in the past. Two of the tested goats had high neutralising antibody titres $\geq 120$. None of the animals were viraemic at the time of testing as all RT-qPCR were conducted according to the standard diagnostic procedures and results from all sera gave negative results.

\section{Ticks and natural foci}

A total of 424 ticks were collected in three sampling activities in July, August and September 2016 along the meadows and in a forest from the vegetation along the daily pathway of the goats from the meadows to the stables. All ticks were morphologically identified as Ixodes ricinus. The single larval pool (17 larvae), the 33 nymphal pools (total 237 nymphs) and all 24 adult male pools (total 81 males) gave negative results in the TBEV RT-qPCR. One of 23 adult female pools (total 89 female ticks), which was sampled on 22 July 2016 and contained two adult females, was PCR-positive for TBEV. Testing the ticks from this pool individually, showed one adult female to be positive for TBE viral genome. An overall infection rate of $0.24 \%$ (1/424) was determined in the local tick population. A phylogenetic comparison of the $E$ genes from the cheese isolates with the $E$ gene sequence of the positive tick showed almost identical sequences. Only 2 of 1,433 nt were different and did not entail amino acid changes (Figure 2).

\section{Discussion}

Milk-borne TBE outbreaks have been reported since the 1950 s in Europe. Transmission of TBEV by milk of goats, sheep and cows is known and was observed in recent decades mainly in central and eastern European countries [7-14]. In Germany, food-borne transmission of TBEV has so far not been reported and is not regarded as epidemiologically important [20]. We report here the simultaneous occurrence of two cases of TBE transmitted by raw goat milk or cheese in Germany. A specific goat farm in south-west Germany could be identified as the location of infection. Although tick bites as source of infection could not be excluded, none of the two cases reported any ticks or tick bites on the suspected day of infection Furthermore, the probability of two concurrent TBE cases occurring during one stay in a TBE focus, assuming an average TBEV prevalence rate in ticks of $0.5 \%$, is minimal.

This cluster of milk-borne TBEV outbreak in Germany had some unusual characteristics. None of more than 20 other guests of the goat farm who were exposed on the same day to the same batches of cheese and milk presented any neurological symptoms resembling TBE. In all milk-borne outbreaks described so far, the manifestation index among exposed and infected persons was high [8-14]. The infectious dose for infection of humans via the oral route by dairy products is unknown. One possible explanation could be that only small portions of the milk or cheese on the goat farm contained enough TBEV to cause human infection. Our RT-qPCR and isolation results led us to conclude that TBEV was inhomogeneously distributed in the goat cheese, which implies that the overall amount of virus in the investigated cheese was small, maybe at the lower range of the oral infectious dose for humans. As we have some indication of infectious raw milk and raw milk cheese from May (human cases) and June (cellculture positive cheese), it is noteworthy that no other human TBE cases could be linked to the consumption of the products.

It is known that low-pathogenic and non-pathogenic TBEV strains circulate in ticks in nature [21]. Therefore, the TBEV isolates from milk and ticks will offer a unique opportunity to study phenotypical and pathogenetic characteristics so as to find first evidence on whether passage through the goat and shedding by goat milk could change the genotype and pathogenicity of TBEV. Data on the pathogenesis of TBE in the gastrointestinal tract are not yet available. It remains unclear where exactly the oral TBEV infection will take place, in the upper or lower gastrointestinal tract. In the latter case, it needs to be studied how TBEV survive the gastric passage with its acid environment.

Our interpretation of the serological results is that one or both goats with the high levels of neutralising TBEV antibodies may have been the source of the infections and might have shed TBEV with milk at the time of infection of the two cases. We do not have any data on the shedding of TBEV in different goat breeds. It is also unknown whether different TBEV strains exhibit different shedding rates in goats. One explanation of the low virus content in the milk products might be that the goat breed in question did not shed large amounts of TBEV in the milk or that the local TBEV strain might not replicate well in goats. We cannot exclude that one or more goats that were shedding virus were re-infected and therefore shedding only small amounts of TBEV in milk. So far, information is scarce on the duration of immunity in goats after TBEV infection, re-infection of previously infected animals and shedding under such circumstances, although one study has suggested immunity in goats after natural infection and vaccination [18]. Recent data demonstrated a decrease in antibodies against TBE in experimentally infected goats 
within 1 year [22]. Only systematic experimental infection of different goat breeds can test this hypothesis.

The detection of TBEV in a tick from the meadows of the farm demonstrates that TBEV circulated in ticks in the region in 2016 . The serological data from the goats imply that some goats had been infected a longer time ago, while two goats with high anti-TBE antibody titres may have had a recent infection. The data suggest that TBEV infection in goats may occur and confirm earlier results from southern Germany [4].

To the best of our knowledge, this is the first time that TBEV could be isolated from cheese of a naturally infected goat after a cluster of TBE with transmission through goat milk. It is so far unclear whether the passage through the goat and the shedding via milk may select for virus strains with changed pathogenicity by the oral route. Our isolates from cheese and from a tick will allow addressing this question by sequencing and analysis of the complete genome of the isolates in ongoing and future studies.

Phylogenetic analysis of the E gene of one of TBEV isolates from goat cheese showed that the virus formed a genetic cluster with TBEV strains from different locations in Switzerland and Austria. This result may support the working hypothesis of discontinuous recent spread of TBEV over long distances, maybe by birds or by human activities (e.g. passive transport by pet animals).

During the 5 years before our cluster (2011-15), 608 human TBE cases were notified in the State of BadenWürttemberg. Only 11 cases were reported from the district where the goat milk transmission occurred (incidence<1:100,000 population) and no cases were reported from the community to which the goat farm belongs. Ca 30-50\% of all notified human TBE cases in Germany do not remember a tick bite before the disease. It has to be studied in more detail whether some of these cases may have been caused by food-borne transmission. We therefore recommend that public health officials and physicians consider this route of infection in the future.

Recent laboratory findings showed that TBEV may survive in processed milk within the food chain [23]. Therefore, the risk of contamination of raw milk products ready for consumption should be reassessed. Until then, consumer protection authorities should provide tailored messages to communicate the risk. This milk-borne TBE cluster in Germany shows the potential of TBEV spread and emergence also in a small local manufacturers even in countries with highly industrialised food production and again illustrates our deficits in the knowledge of TBEV pathogenicity and eco-epidemiology.
${ }^{*}$ Addendum

The GenBank accession numbers were added on 1 October 2018 in the note to Figure 2.

Conflict of interest

None declared.

\section{Authors' contributions}

Brockmann Stefan: Head and coordinator of Outbreak investigation. Oehme Rainer: Testing of milk samples and ticks; isolation of one TBEV virus strain. Buckenmaier Thomas: Veterinary outbreak investigation. Beer Martin: Serological testing of goat sera. Jeffery-Smith Anna: Virological diagnosis of index case. Spannenkrebs Monia: Outbreak investigation. Haag-Milz Susanne: Outbreak investigation. Wagner Wiening Christiane: Registration of human cases on State Level. Schlegel Christiane: Outbreak investigation. Fritz Jana: outbreak investigation, registration of human cases on district level. Zange Sabine: Virological confirmation of human cases. Bestehorn Malena: Phylogenetic analysis of TBEV strains. Lindau Alexander: Sampling and testing of ticks. Hoffmann Donata: serological testing of goat sera. Tiberi Simon: Treatment and patient care of index case. Mackenstedt Ute: coordinator of tick sampling and testing. Dobler Gerhard: virus isolation of one TBEV strain from goat cheese; coordination of all activities of outbreak investigation.

\section{References}

1. Lindquist L, Vapalahti O. Tick-borne encephalitis. Lancet. 2008;371(9627):1861-71. https://doi.org/10.1016/S01406736(08)60800-4 PMID: 18514730

2. Bogovic P, Strle F. Tick-borne encephalitis: A review of epidemiology, clinical characteristics, and management. World J Clin Cases. 2015;3(5):430-41. https://doi.org/10.12998/wjcc. v3.i5.430 PMID: 25984517

3. Klaus C, Hoffmann D, Hoffmann B, Beer M. FrühsommerMeningoenzephalitis-Virus-Infektionen bei Tieren - Klinik, Diagnostik und epidemiologische Bedeutung.[Tick-borne encephalitis infections in animals - clinical symptoms, diagnostics and epidemiological relevance]. Berl Munch Tierarztl Wochenschr. 2016; advance online publication. German. https://doi.org/10.2376/0005-9366-16062

4. Klaus C, Beer M, Saier R, Schau U, Moog U, Hoffmann B, et al. Goats and sheep as sentinels for tick-borne encephalitis (TBE) virus--epidemiological studies in areas endemic and non-endemic for TBE virus in Germany. Ticks Tick Borne Dis. 2012;3(1):27-37. https://doi.org/10.1016/j.ttbdis.2011.09.011 PMID: 22309856

5. Gresíková M, Sekeyová M, Stúpalová S, Necas S. Sheep milk-borne epidemic of tick-borne encephalitis in Slovakia. Intervirology. 1975;5(1-2):57-61. https://doi. org/10.1159/000149880 PMID: 1237478

6. Amicizia D, Domnich A, Panatto D, Lai PL, Cristina ML, Avio $\mathrm{U}$, et al. Epidemiology of tick-borne encephalitis (TBE) in Europe and its prevention by available vaccines. Hum Vaccin Immunother. 2013;9(5):1163-71. https://doi.org/10.4161/ hv.23802 PMID: 23377671

7. Gresikova M, Rehacek J. Isolierung des Zeckenenzephalitisvirus aus Blut und Milch von Haustieren (Schaf und Kuh) nach Infektion durch Zecken der Gattung Ixodes ricinus L. [Isolation of the tick encephalitis virus from blood ad milk of domestic animals (sheep and cow) after infection by ticks of the family Ixodes ricinus L.]. Arch Gesamte Virusforsch. 1959;9(3):3604. German. https://doi.org/10.1007/BF01248828 PMID: 13829212

8. Gresikova M. [The transmission of the virus of the Czechoslovak tick encephalitis by goat milk]. In: Zeckenenzephalitis in Europa [Tick-borne encephalitis in Europe]. Libikova $\mathrm{H}$, editor. Abhandlungen der Deutschen Akademie der Wissenschaften zu Berlin. Akademieverlag Berlin. Vol 1960 (2):121-2. 
9. Kríz B, Benes C, Daniel M. Alimentary transmission of tick-borne encephalitis in the Czech Republic (1997-2008). Epidemiol Mikrobiol Imunol. 2009;58(2):98-103. PMID: 19526924

10. Cisak E, Wójcik-Fatla A, Zając V, Sroka J, Buczek A, Dutkiewicz J. Prevalence of tick-borne encephalitis virus (TBEV) in samples of raw milk taken randomly from cows, goats and sheep in eastern Poland. Ann Agric Environ Med. 2010;17(2):283-6. PMID: 21186771

11. Caini S, Szomor K, Ferenczi E, Szekelyne Gaspar A, Csohan A, Krisztalovics K, et al. Tick-borne encephalitis transmitted by unpasteurised cow milk in western Hungary, September to October 2011. Euro Surveill. 2012;17(12):20128. PMID: 22490310

12. Hudopisk N, Korva M, Janet E, Simetinger M, Grgič-Vitek M, Gubenšek J, et al. Tick-borne encephalitis associated with consumption of raw goat milk, Slovenia, 2012. Emerg Infect Dis. 2013;19(5):806-8. https://doi.org/10.3201/eid1905.121442 PMID: 23697658

13. Markovinović L, Kosanović Ličina ML, Tešić V, Vojvodić D, Vladušić Lucić I, Kniewald T, et al. An outbreak of tick-borne encephalitis associated with raw goat milk and cheese consumption, Croatia, 2015. Infection. 2016;44(5):661-5. https://doi.org/10.1007/s15010-016-0917-8 PMID: 27364148

14. Holzmann H, Aberle SW, Stiasny K, Werner P, Mischak A, Zainer $B$, et al. Tick-borne encephalitis from eating goat cheese in a mountain region of Austria. Emerg Infect Dis. 2009;15(10):16713. https://doi.org/10.3201/eid1510.090743 PMID: 19861072

15. Robert Koch Institut (RKI). SurvStat@RKI 2.o. Berlin: RKI. [Accessed: 2 May 2017]. https://survstat.rki.de/

16. Schwaiger M, Cassinotti P. Development of a quantitative real-time RT-PCR assay with internal control for the laboratory detection of tick borne encephalitis virus (TBEV) RNA. J Clin Virol. 2003;27(2):136-45. https://doi.org/10.1016/S13866532(02)00168-3 PMID: 12829035

17. Kupča AM, Essbauer S, Zoeller G, de Mendonça PG, Brey $R$, Rinder $M$, et al. Isolation and molecular characterization of a tick-borne encephalitis virus strain from a new tickborne encephalitis focus with severe cases in Bavaria, Germany. Ticks Tick Borne Dis. 2010;1(1):44-51. https://doi. org/10.1016/j.ttbdis.2009.11.002 PMID: 21771510

18. Klaus C, Beer M, Saier R, Schubert H, Bischoff S, Süss J. Evaluation of serological tests for detecting tick-borne encephalitis virus (TBEV) antibodies in animals. Berl Munch Tierarztl Wochenschr. 2011;124(11-12):443-9. PMID: 22191165

19. Gäumann R, Mühlemann K, Strasser M, Beuret CM. Highthroughput procedure for tick surveys of tick-borne encephalitis virus and its application in a national surveillance study in Switzerland. Appl Environ Microbiol. 2010;76(13):4241 9. https://doi.org/10.1128/AEM.00391-10 PMID: 20453126

20. Rieger MA, Nübling $M$, Kaiser R, Tiller FW, Hofmann F. FSMEInfektionen durch Rohmilch--welche Rolle spielt dieser Infektionsweg? Untersuchungen aus dem südwestdeutschen FSME-Endemiegebiet. Gesundheitswesen. 1998;60(6):348-56. PMID: 9697358

21. Dobler G, Bestehorn M, Antwerpen M, Överby-Wernstedt A. Complete genome sequence of a low-virulence tick-borne encephalitis virus strain. Genome Announc. 2016;4(5):e0114516. https://doi.org/10.1128/genomeA.01145-16 PMID: 27795275

22. Salat J, Slosarkova S, Ruzek D, Hunady M. Tick-borne encephalitis: The risk of alimentary infection of small ruminants in the Czech Republic. Poster presented at: 9th Conference on Ticks and Tick-borne Pathogens; 27 Aug-1 Sep 2017; Cairns, Australia.

23. Saier R, Hinrichs J. FSME-Antikörper-Vorkommen bei Ziegen aus Baden-Württemberg und Untersuchungen zur thermischen Stabilität von FSME-Erregern in Milch [Occurrence of TBE antibodies in goats from Baden-Württemberg and studies on the thermal stability of TBE viruses in milk]. German. Presented at: 2. Süddeutscher Zeckenkongress; 17-18 Mar 2014; Stuttgart, Germany.

\section{License and copyright}

This is an open-access article distributed under the terms of the Creative Commons Attribution (CC BY 4.0) Licence. You may share and adapt the material, but must give appropriate credit to the source, provide a link to the licence, and indicate if changes were made.

This article is copyright of the authors, 2018. 